\title{
Dynamic Channel Assignment algorithms for supporting QoS based services in wireless cellular environments
}

\author{
P.M.Papazoglou ${ }^{1}$, D.A.Karras ${ }^{2}$, and R.C.Papademetriou ${ }^{3}$
}

\begin{abstract}
The number of users employing multimedia services in cellular networks has been increased dramatically in the last decade. Multimedia services are more demanding compared to traditional voice services in terms of bandwidth availability and integrity. The capacity and the bandwidth reservation on demand according to QoS requirements are key concepts in mobile cellular communications. The available frequency spectrum is limited and the demand for enhanced mobile user facilities is increasing day by day, hence the channels must be reused as much as possible to increase the system capacity. Existing and proposed channel assignment strategies have to be transformed to support efficiently the new demanding multimedia services. Through this paper we present a review of the most known channel assignment strategies, as well as their QoS aspects and we propose new DCA variations and a channel architecture adapted to QoS requirements for supporting multimedia services. We also present some strategies, models and conclusions for algorithm evaluation based on experimental results.
\end{abstract}

Keywords - Wireless networks, Cellular networks, Dynamic Channel Allocation, QoS services, Multimedia Services.

\section{INTRODUCTION}

The cellular principle divides the covered geographical area into a set of smaller service areas called cells. In order to establish a communication with the base station of a cell, a mobile terminal must first obtain a channel from this base station. A channel consists of a pair of frequencies (uplink/downlink) for bidirectional communication. The capacity of a cellular system can be described in terms of the number of available channels, or the number of users the system can support. The total number of channels made available to a system depends on the allocated spectrum and the channel bandwidth. The available frequency spectrum is limited and the number of mobile users is increasing, hence the channels must be reused as much as possible to increase the system capacity. The role of a channel assignment scheme is to allocate channels to cells or mobiles so as to minimize: a) the probability that the incoming calls are dropped, b) the probability that ongoing calls are dropped, and c) the probability that the carrier-to-interference ratio of any call falls below a fixed value.

\footnotetext{
Sterea Hellas Institute of Technology, Greece

2 Sterea Hellas Institute of Technology, Dept. Automation, Chalkis, P.C 34400, Greece, e-mails: dakarras@teiste.gr, dimitrios.karras@gmail.com,dakarras@ieee.org

${ }^{3}$ Dept. Electronics and Computer Engineering, Univ. of Portsmouth, Anglesea Road, Portsmouth, PO1 3DJ UK
}

\section{QOS (QUALITY OF SERVICE) IN WIRELESS NETWORKS}

The user demand for advanced mobile services such as data transfer, video telephony, internet access, multimedia applications, etc, bring out much more the problem of the available bandwidth. Algorithm design for efficient bandwidth management is a critical issue for multimedia cellular network design. Quality of service (QoS) can be studied from many different points of view. In cellular communications the bandwidth management is the key concept for achieving the desired QoS. Many bandwidth management strategies have been proposed such us bandwidth reservation, reducing handoff dropping probability $[1,2,3]$,etc.

Multimedia applications can be classified into real-time and non-real-time based on delay requirements. Advanced applications such as video conference, etc have stringent timing constraints and so the delay tolerance is very small. A resource management system has to utilize efficiently the system resources and provide prioritized user treatment based on users traffic profile especially when the cell or network is congested in order to meet bandwidth requirements.. Channel assignment strategies are the key for efficient capacity management. The selected strategy has to guarantee the continuity of a multimedia ongoing call in hand-off or in weak signal conditions offering channel capacity within the delay tolerant limits. Call admission priorities can be set based on user payment or call grandness. Bandwidth reservation is also a very popular methodology for QoS support.

\section{Channel Allocation Strategies}

The channel assignment schemes in general can be classified into three strategies: Fixed Channel Assignment (FCA) [4,5,6,7,8], Dynamic Channel Assignment (DCA) [4,9,10,11,12], and the Hybrid Channel Assignment (HCA) [4,13]. In FCA, a set of channels are permanently allocated to each cell based on a pre-estimated traffic intensity. In DCA, there is no permanent allocation of channels to cells. Rather, the entire set of available channels is accessible to all the cells, and the channels are assigned on a call-by-call basis in a dynamic manner. One of the objectives in DCA is to develop a channel assignment strategy, which minimizes the total number of blocked calls. The FCA scheme is simple but does not adapt to changing traffic conditions and user distribution. To overcome the drawbacks of FCA and DCA, HCA was proposed by Kahwa et al.[4]. DCA schemes can be centralized or distributed. In the centralized approach [4] all requests for channel allocation are forwarded to a channel controller that has access to system wide channel usage information. In 
distributed DCA [9], the relevant decision is taken by the concerned base station on the basis of the information from the surrounding cells.

\section{QoS (Quality of Service) assurance}

Required channel capacity based on services constraints Each user service requires a different bit rate in relation with bandwidth and signal to noise -plus- interference ratio. Channel capacity can be calculated using the known Shannon-Hartley formula. The needed channel capacity must be calculated according to the channel type that is based on service category. Mobile services can be divided in three categories which are : a) Voice channels, b) Video channels and c) Data channels. The Data channels are categorized in various classes based on the amount of data to be transferred. QoS can be achieved using specific criteria in relation with the selected DCA schemes. The basic proposed QoS measures are: Service capacity, Delivery time, Forced call probability and Call Admission delay. In this work we have explicitly exploited service capacity but one could generalize involving the other measures too in the proposed algorithms.

When current service capacity $\geq$ capacity threshold, then the QoS is acceptable. If we assume that each channel has a bandwidth of $\mathrm{n} \mathrm{KHz}$ for example, then the selected DCA variation algorithm has to guarantee the required signal to noise -plus- interference ratio. The second criterion is the delivery time and constitutes a combination of time and channel capacity. Forced call probability represents the call service continuity against user movement and undesired signal strength. Finally, the last criterion represents the call admission delay due to bandwidth unavailability.

\section{Channel Bandwidth Architecture}

Each network cell provides with communication channels the candidate mobile users. The channel stack is the main idea for the proposed bandwidth usage architecture. In cellular network a different bandwidth exists for the uplink and downlink respectively.

Our algorithm faces the uplink and downlink bandwidths as a dynamically changing channel stack. This stack consists of two operational regions. One with fixed width channels and one with variable width channels (fig. 1). The fixed region serves the voice calls and the variable the advanced calls (video, data transfer, etc).

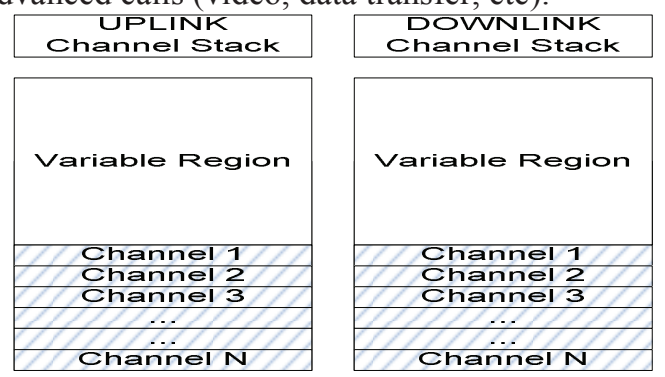

Figure 1. Channel Stack/ Channel Model

\section{PROPOSED DCA VARIATIONS WITH QOS SUPPORT}

The basic feature of the bandwidth management algorithm must be the adaptability to the current network traffic conditions. The proposed bandwidth management algorithm is not restricted only in handoff management like other studies do [1,2,3]. Our proposed algorithms constitute a whole system that manages all the basic network procedures such as new call arrival, reallocation, call termination etc, in order to achieve higher adaptability to user needs and traffic conditions as originally presented in [14] for voice only calls in a GSM network. However, Multimedia services require a more complex approach involving variable size channels and a different channel model based on bandwidth assignments and relevant QoS threshold satisfaction, not simple fixed channels as in the voice case of [14]. The main concepts of the herein novel proposed algorithms are:

- Bandwidth management for all the network procedures, not only for handoff

- Channel stack for the bandwidth usage architecture

- Algorithms that are based mainly on CNIR (Carrier to Noise and Interference Ratio) factor

- Bandwidth compression (if needed) for non real time services

- Reallocation of ongoing services when the remaining time is below a threshold in order to minimize the cell/bandwidth congestion

We have used our simulation system to evaluate the new proposed DCA variations which are mainly:

- Balanced version (based on least-congested algorithm around neighbors)

- Best CNR (Carrier to Noise) version (based on strongest CNR around neighbors)

- Round blocking version (based on unbalanced DCA version around neighbors)

Firstly, the algorithm tries to assign a channel within the initiated cell of the new call and afterwards activates any of the above methods.

The above mentioned procedures such as reallocation, new call arrival and user movement are adapted each time to the selected DCA variation (e.g. balanced, unbalanced, round blocking, etc)

\section{Balanced variation}

The DCA unbalanced algorithm assigns the first channel that fulfills the conditions and the DCA balanced uses the least congestion algorithm for the completion of channel assignment.

As we mentioned before, the balanced method uses the least congested algorithm. Initially, we assume that the least congested cell is the cell where the new call is occurred. The algorithm scans all the cells in the neighbor region and finds the least congested cell (figure 2a).

\section{Best CNR (Carrier to Noise Ratio) variation}

The best CNR method goes around the six neighbor cells of the initiated cell and calculates the CNR between user and neighbor cell base stations. After the strongest CNR is found, the program tries to assign a new channel from the corresponding cell. The algorithm initially assumes that the strongest CNR exists from the first neighbor cell and continues to calculate CNR from the rest neighbor cells until the strongest CNR is found. Figure $2 b$, illustrates the algorithm structure.

The computations are based on signal strength and how is affected from other connected users in neighbor cells. If a 
user signal does not fulfill the CNIR, that is $\mathrm{C} /(\mathrm{N}+\mathrm{I})$, threshold (Carrier to Noise and Interference ratio) the procedure tries to find another appropriate channel. The Carrier to Noise and Interference ratio can be derived using the type [1]-[3]

$$
R_{c n i}=\frac{A P_{0} d_{0}^{-\alpha} 10^{\frac{\xi_{0}}{10}}}{N+\sum_{i=1}^{n} A P_{i} d_{i}^{-\alpha} 10^{\frac{\xi_{i}}{10}}}
$$

Where $\mathbf{n}$ is the number of base stations and users, $\mathbf{d}_{\mathbf{i}}$ is the distance between user and base station, $\boldsymbol{\xi}_{\mathrm{i}}$ is the distortion due to shadowing from user to base station, $\mathbf{A}$ is a proportional coefficient and $\mathbf{P}$ is the transmitted power.

\section{Round Blocking variation}

The round blocking algorithm searches in neighbor cells and when a successful channel assignment is made the algorithm stops. If the call establishment is not successful in the last neighbor cell, the call is blocked.

\section{EXPERIMENTAL RESULTS}

We have developed a complete simulation environment for the evaluation of the proposed channel assignment algorithms under QoS considerations. Through this environment we produce some important statistical metrics such as blocking probability, dropping probability, cell congestion, channel reusability, etc for the behavior of the designed cellular network. Our experimental results comprise performance graphs for the proposed DCA variations for supporting classical GSM voice services as well as performance graphs of the transformed DCA variations for supporting sample multimedia services. Each user entering the wireless network in our simulations is assigned a random communication load for mixed voice/video/data services. Voice needs one fixed channel only, for each user, while video and data services need a number of such fixed channels according to the necessary bandwidth assigned randomly to each user,, complying with channel stack model architecture of figure 1. That is, for video and data each user needs a variable number of fixed channels (each one with equal capacity to the voice channel) $=$ integer part of the division [user necessary bandwidth / fixed channel capacity]

The simulation results include data for calculating important measures such as blocking probability, dropping probability, channel reuse, cell congestion, number of reallocations, channel reuse per cell, number of new calls in simulation time, etc. The generated matlab files include additional command for calculating some statistical metrics of simulation results like Standard deviation, Covariance and Average value.

\section{DCA comparative results.}

We have simulated a cellular network of 19 cells capacity with $10,50,80,100,200,400,750$ users per cell and 32 channels per cell. The channel allocation schemes that have been used are Centralized DCA (Balanced, Unbalanced with random call arrival and random/Gaussian user movement) and distributed DCA (Balanced, Unbalanced, round blocking, best CNR with Poisson call arrival and Gaussian user movement).

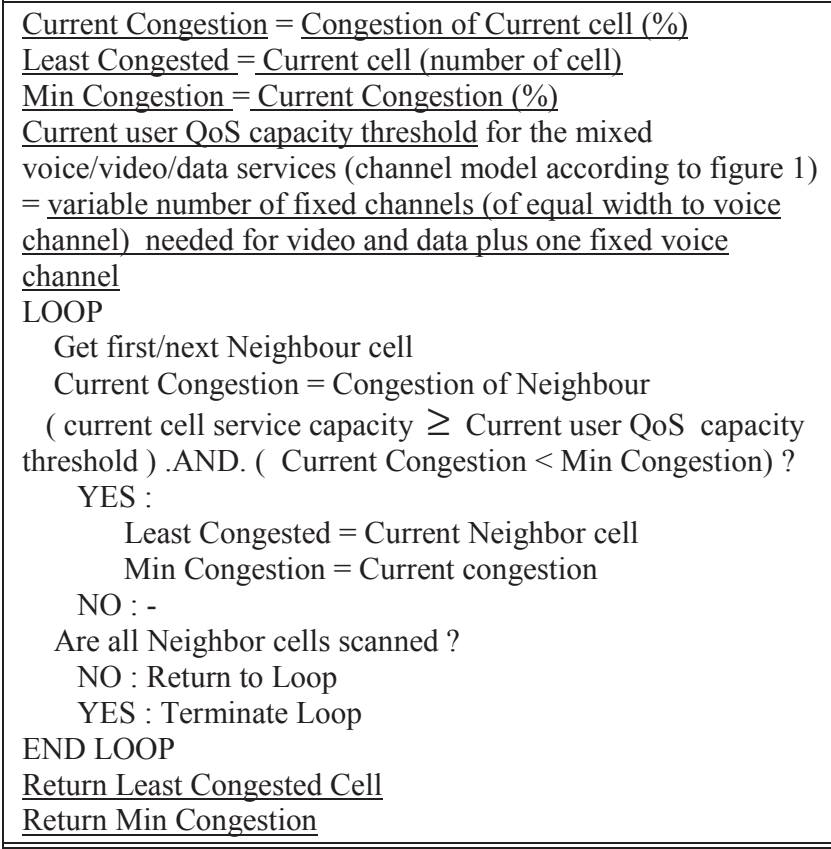

Figure 2a - The least congestion algorithm (balanced variation)

Max CNR cell $=$ First Neighbour cell

$\underline{\text { Current cell }}=\underline{\text { First Neighbour cell }}$

Max CNR $=$ CNR of Current cell

Current user QoS capacity threshold for the mixed

voice/video/data services (channel model according to figure 1)

$=$ variable number of fixed channels (of equal width to voice

channel) needed for video and data plus one fixed voice channel

LOOP

Get first/next Neighbour cell

Current cell $=$ Neighbour cell

Compute CNR for Current cell

( current cell service capacity $\geq$ Current user QoS capacity threshold ).AND. (Current CNR $>$ Max CNR )?

YES :

Max CNR cell $=$ Current Neighbour cell

Max CNR $=$ CNR of Current Neighbour cell NO :

Are all Neighbour cells scanned?

NO : Return to Loop

YES : Terminate Loop

END LOOP

Return Max CNR Cell

Figure 2b - The Best CNR algorithm

Figure 3 illustrates the performance in terms of blocking probability of the main proposed DCA variations for voice only call services in the classical GSM environment.

Figures 4 and 5 illustrate the performance of the wireless network in terms of blocking and dropping probability with regards to the main proposed DCA variations for a mixture of communication load services including voice, video and data services in GSM environment. Regarding figure 4, no user movement is assumed, while in figure 5 poisson call arrival and Gaussian user movement are assumed. From these graphs it seems that best CNR and round blocking variations perform better than the balanced one. However, the balanced variation proves to be always better than the traditional unbalanced DCA scheme. 
Because of the algorithms structure in Figures 2a, 2b it is obvious that in order to satisfy (in the LOOPS of these figures) the Current user QoS capacity threshold, it is possible not to be able to assign bandwidth to the newcomer users of these simulated multimedia GSM networks. Thus, the blocking probabilities increase for the newcomers, while dropping probabilities, for the already bandwidth assigned users could be more stable or decreasing.

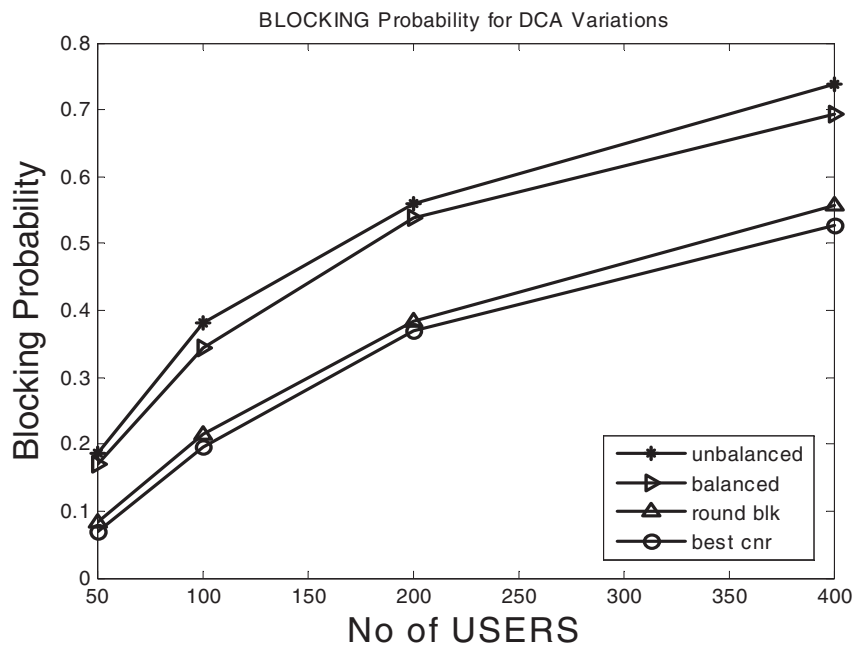

Figure 3. Blocking probability for voice only communications

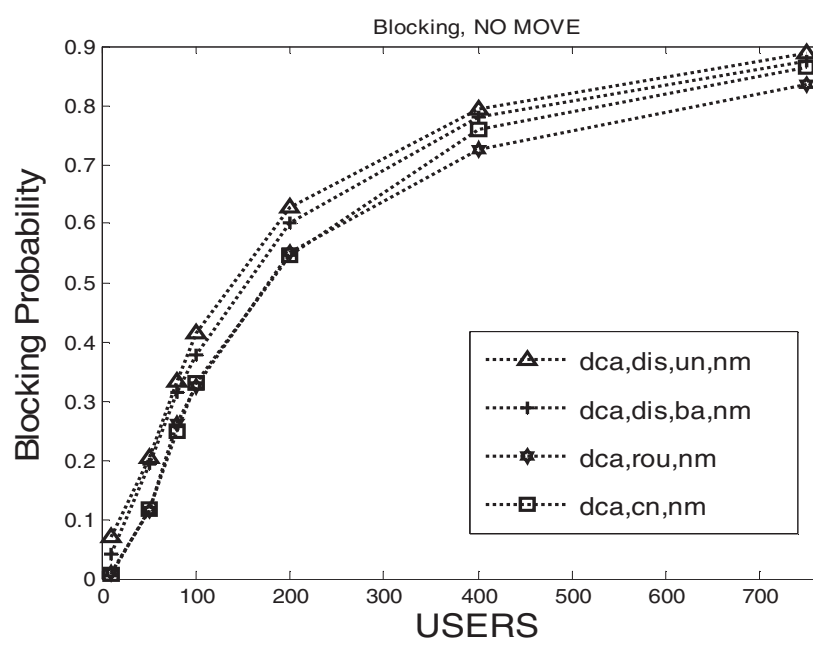

Figure 4. Blocking probability of the proposed DCA variations (poisson call arrival, NO user movement), for voice/video/data (each user is initiated with voice plus random load for video and data) communications (un=unbalanced/ $\mathrm{ba}=$ balanced/rou=round blocking $/ \mathrm{cn}=$ best $\mathrm{CNR}$ ).

\section{CONCLUSIONS AND FUTURE PROSPECTS}

In this research paper we have presented a variation of the channel model architecture in wireless networks simulation systems for involving multimedia services and QoS requirements in the channel assignment problem. Under these considerations we have proposed variations of channel assignment strategies for the traditional DCA scheme, as well as their QoS aspects for supporting multimedia services for the mobile users. We also present some strategies, models and conclusions for algorithm evaluation based on experimental results. These results are very promising and need to be extended in large scale real world wireless systems

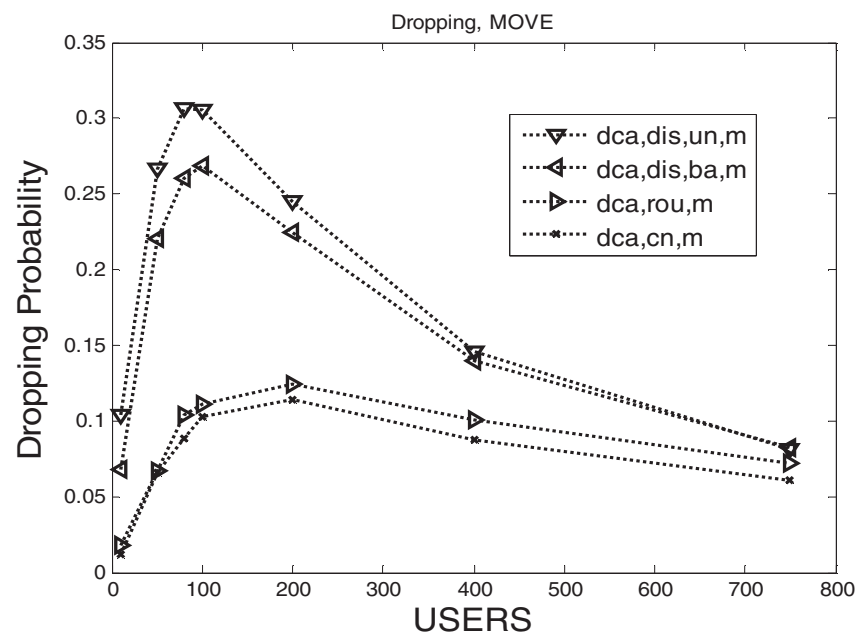

Figure 5 - Dropping probability of the proposed methods poisson call arrival, Gaussian user movement, for voice/video/data (each user is initiated with voice plus random load for video and data) communications (un=unbalanced/ $\mathrm{ba}=$ balanced/ rou=round blocking/ $\mathrm{cn}=$ best $\mathrm{CNR}$ ).

\section{REFERENCES}

[1] S. Kim and P.K.Varshney, "An Integrated Adaptive BandwidthManagement Framework for QoS-Sensitive Multimedia Cellular Networks", Transactions of IEEE on Vehicular technology, Vol.53, No.3,May 2004

[2] H. B. Kim, "An Adaptive Bandwidth Reservation Scheme for Multimedia Mobile Cellular Networks", In Proc. of IEEE Intern. Conf. on Communications (ICC 2005), Seoul, Korea, May 2005.

[3] N. Nasser and H. S. Hassanein, "Bandwidth Reservation Policy for Multimedia Wireless Networks and its Analysis," IEEE International Conference on Communications, June 2004.

[4] M.Zhang, and T. S. Yum, "Comparisons of Channel Assignment Strategies in Cellular Mobile Telephone Systems", IEEE Trans. on Vehicular Technology, vol.38,no.4,pp211-215,1989.

[5] W.K. Lai and G.C. Coghill, "Channel Assignment through Evolutionary Optimization”, IEEE Transactions on Vehicular Technology, vol.45,no.1,pp91-96,1996.

[6] Ye, Qiaoyang, et al. "User association for load balancing in heterogeneous cellular networks." Wireless Communications, IEEE Transactions on 12.6 (2013): 2706-2716.

[7] Golrezaei, Negin, et al. "Femtocaching and device-to-device collaboration: A new architecture for wireless video distribution.", Communications Magazine, IEEE 51.4 (2013): 142-149.

[8] Goyal, Ankit, and Sanjiv Kumar Tomar. "GBA-An Efficient Method for Fixed Channel Allocation in Cellular Network." International Journal of Advanced Research in Computer Science 3.3 (2012).

[9] Mishra, M. P., and P. C. Saxena. "Survey of channel allocation algorithms research for cellular systems." Int J Netw Commun 2.5 (2012): 75-104.

[10] Viswanadh, K., and Dr G. Rama Murthy. "Optimal Hybrid Channel Allocation: Based On Machine Learning Algorithms." arXiv preprint arXiv:1309.7439 (2013).

[11]Wang, Chiapin, and Wen-Hsing Kuo. "A utility-based resource allocation scheme for IEEE 802.11 WLANs via a machine-learning approach.", Wireless Networks 20.7 (2014): 1743-1758.

[12] Singh, Deepak Kumar, K. Srinivas, and D. Bhagwan Das. " $A$ dynamic channel assignment in GSM telecommunication network using modified genetic algorithm." Proceedings ,6th Euro American Conference on Telematics and Information Systems. ACM, 2012.

[13] Feng, Daquan, et al. "A survey of energy-efficient wireless communications.", Communications Surveys \& Tutorials, IEEE 15.1 (2013): 167-178.

[14] Papazoglou, P.M., D.A. Karras, and R.C. Papademetriou, "Evaluating novel DCA variations for efficient channel assignment in cellular communications through a generic Java simulation system", Proceedings of 2011 Intern. Conference on Computer Science and Network Technology, Harbin, 24-26 Dec, 2011. 